\title{
Conceptual cueing and the recall of order in threaded lists'
}

\author{
G. J. HARRISON \\ UNIVERSITY OF SHEFFIELD, ENGLAND
}

The immediate recall of all the (known) items of lists in their presented order was compared with the recall of cued items from lists: specifications of relevant items sticceeded presentation. In partial recall category names cued recall of category members. Members of each category were distributed throughout lists; even with these lists, recall in the partial condition exceeded that in the total condition.

In an earlier study (Harrison, 1964) it was found that the recall of parts of a list specified after presentation was proportionately greater than the recall of complete lists. The lists used had always contained the names of the four cardinal points of the compass, the names of the four gospel writers, and the names of the card suits. Further, the members of a category always occurred consecutively. Hence, one can ask if the observed improvement in partial recall might be ascribed to having cued partial recall by naming a category whose members appeared consecutively. The reason for this inquiry is the notable component of the partial recall improvement obtained on cueing the category forming the last third of a list for partial recall. A large improvement in partial recall for the final third of a 12 digit list was reported by Anderson (1960) in her study of the influence of responding in short-term retention. One way to inquire into the relative roles of responding and recency in producing the proportionate superiority of partial recall is to present threaded lists. If the partial recall improvement is mainly due to a recency effect it should be drastically reduced by using threaded lists such as: Mark CLUBS North Matthew DIAMONDS East John HEARTS South Luke SPADES West.

\section{Method and Procedure}

Eighteen threaded lists were recorded at a $1 \mathrm{sec}$ rate. Nine were for partial recall and nine for total. Each list from one of these sets corresponded with one from the other set in its structure. Suppose, for example, in one partial recall list the order of use of items from the three categories was one of the gospel writers, one from the card suits, and one from the compass points-this being repeated through the list as in the above illustration. To correspond with this list for partial recall there would be a similar one cued for total recall. Suppose, too, that the cue following the partial recall example was "Suits." Then in both the partial recall list and the corresponding total list only the responses ordering the names of the card suits would be relevant in scoring.
Hence the maximum score for both lists would be 4 , while over the whole experiment maximum scores for the two recall conditions would be 36 .

The three cues appended to lists for partial recall were "Compass," Gospels," and "Suits." The cue for total recall was "All." Cues followed $1 \mathrm{sec}$ after the final item of the lists.

Subjects were told of the items to appear in the lists and of the relevance of the cues. They attempted recall of two practice lists-one cued for partial and one for total recall. When the task was understood, the experimental lists were presented. The recall intervals were of $15 \mathrm{sec}$ for partial recall lists and $45 \mathrm{sec}$ for total recall ones. Responses were transcribed as they were said.

\section{Results}

Table 1 shows performance according to three different measures: (1) Score 1-the total number of relevant items given in the same position at recall as at presentation; (2) Score 2-the number of times in which the above form of partial recall score for individual lists exceeded the score for the corresponding total recall list (P-T positive), and the number of times the reverse was found (P-T negative); (3) Score 3-the number of times in which all four items of a relevant category were correctly ordered in recall for the two conditions.

With eight $\mathrm{Ss}$, those indices showed recall to be significantly better with partial recall $(p=0.035$ on a one-tailed sign test). A two-way analysis of variance was carried out using the data of Score 1 from the present results and corresponding data from the earlier study. This analysis confirmed the significance of the appropriate difference $(F=7.81$, df $=2 / 14, p<0.025$ ) earlier assessed by a nonparametric

Table 1. Scores for the recall of threaded lists

\begin{tabular}{lrccccc} 
Subject & \multicolumn{2}{c}{ Score 1 } & \multicolumn{2}{c}{ Score 2 } & \multicolumn{2}{c}{ Score 3 } \\
& Partial & Total & $\begin{array}{c}\text { P.T } \\
\text { (positive) }\end{array}$ & $\begin{array}{c}\text { P.T } \\
\text { (negative) }\end{array}$ & & \\
\hline J.L. & 22 & 9 & 6 & 1 & 3 & 0 \\
D.M. & 8 & 11 & 3 & 4 & 0 & 1 \\
R.S. & 12 & 9 & 4 & 3 & 0 & 0 \\
L.P. & 16 & 9 & 5 & 2 & 1 & 0 \\
B.T. & 12 & 10 & 3 & 2 & 2 & 0 \\
J.B. & 22 & 15 & 6 & 0 & 2 & 0 \\
J.W. & 19 & 13 & 4 & 2 & 2 & 0 \\
P.H. & 17 & 11 & 4 & 3 & 3 & 1 \\
Totats & 128 & 87 & 35 & 17 & 13 & 2 \\
Mean & 16.00 & 10.875 & 4.375 & 2.125 & 1.625 & 0.25 \\
Medians 16.5 & 10.5 & 4.0 & 2.0 & 2.0 & 0.0 \\
\hline
\end{tabular}


test. It also showed that overall performance with threaded lists was significantly $(\mathrm{F}=24.6, \mathrm{df}=1 / 14$, $p<0.001$ ) inferior to that with lists in which items of a category were presented consecutively. The lists by recall condition interaction was not significant $(F=2.84, \mathrm{df}=1 / 14, p>0.10)$. That is, the extent of the improvement in partial recall did not significantly differ for the two types of list.

By contrast with the previous study it was apparent that Ss did not always make the maximum number of responses to a cue (four for the partial and 12 for the total condition). Analysis showed that in the present study the proportion of responses that were made to those that could be made was significantly greater $(p<0.01)$ in the partial condition. However, the difference between the proportions of correct responses to those actually made did not differ between conditions, being $128 / 272=0.470$ in the partial condition and $87 / 165=0.527$ in the total.

\section{Discussion}

The main question in mind when undertaking this experiment was: Would the improvement in partial recall be drastically reduced when threaded lists were used? The absence of a significant interaction between type of list and recall condition implies a negative answer to this question. Admittedly the change in Ss' strategies complicates assessment of the point, as does the general fall in recall with threaded lists-a less tractable form of material. However, even with threaded lists a significant improvement is found-even using the same small number of Ss as in the earlier (1964) study. Additionally, the ratio of correctly ordered items in the two recall conditions is strikingly similar in both studies. Overall these proportions are 0.679 and 0.639 for the threaded and consecutive lists, respectively. The difference between the two sets of eight such ratios provided by individual $S$ in the two studies was tested using a Mann-Whitney $U$ test, and did not approach significance $(p=0.480)$. With these data it seems reasonable to accept that the superior partial recall of serial lists with known items in varying order cannot be mainly attributed to a recency effect. This exclusion supports the views of Anderson (1960) and later workers, e.g., Waugh \& Norman (1965), that an account of short-term forgetting phrased only in terms of decay is inadequate.

\section{References}

ANDERSON, N. Post stimulus cueing in immediate memory. $J$. exp. Psychol 1960, 60, 216-221.

HARRISON, G. J. Effect of conceptual cueing on the recall of order. Nature 1964, 202, 1365.

WAUGH, N. C., \& NORMAN, D. A. Primary memory. Psych. Rev. 1965, 89-104.

Note

1. This work was supported by a grant from the Social Science Research Council. 